\title{
Cytokines and Inflammation in Meniere Disease
}

\author{
Lidia Frejo $0^{1,2,3}$ (D) $\cdot$ Jose Antonio Lopez-Escamez ${ }^{1,2,3,4}$ (B) \\ ${ }^{I}$ Otology and Neurotology Group CTS495, Department of Genomic Medicine, Centre for Genomics and Oncological Research (GENYO), \\ Pfizer-University of Granada-Junta de Andalucia, PTS, Granada, ${ }^{2}$ Department of Otolaryngology, Instituto de Investigación Biosanitaria ibs. \\ Granada, Hospital Universitario Virgen de las Nieves, Granada; ${ }^{3}$ Sensorineural Pathology Programme, Centro de Investigación Biomédica en \\ Red en Enfermedades Raras, CIBERER, Madrid; ${ }^{4}$ Department of Surgery, Division of Otolaryngology, Universidad de Granada, Granada, Spain
}

Meniere disease (MD) is a rare set of conditions associated with the accumulation of endolymph in the cochlear duct and the vestibular labyrinth with a decrease of endocochlear potential. It is considered a chronic inflammatory disorder of the inner ear with a multifactorial origin. The clinical syndrome includes several groups of patients with a core phenotype: sensorineural hearing loss, episodes of vertigo, and tinnitus with a non-predictable course. Genetic factors and the innate immune response seem to play a central role in the pathophysiology of the condition. Autoimmune MD should be diagnosed if a patient fulfills the diagnostic criteria for $\mathrm{MD}$ and one of the following autoimmune disorders: autoimmune thyroid disease, psoriasis, autoimmune arthritis, ankylosing spondylitis, or systemic lupus erythematosus. We summarize the evidence to support autoimmune MD as an endophenotype in bilateral MD associated with the allelic variant rs4947296 and nuclear factor-kappa B (NF-kB)-mediated inflammation, the role of cytokines (particularly interleukin-1 $\beta$ and tumor necrosis factor- $\alpha$ ) in defining a subset of patients with autoinflammation, and the potential role of cytokines as biomarkers to distinguish between patients with MD and vestibular migraine. Finally, we also introduce a list of potential drugs that could regulate the immune response in $\mathrm{MD}$ with potential for repurposing in clinical trials.

Keywords. Meniere Disease; Cytokines; Autoimmunity; Autoinflammatory Disease; Vestibular Disease

\section{INTRODUCTION}

Meniere disease (MD) was first described by Prosper Ménière in 1861. It is a multifactorial inner ear disorder, the onset and progression of which are triggered by the combined effects of genetic, epigenetic, and environmental factors. MD is characterized by recurrent episodes of vertigo associated with ipsilateral cochlear symptoms occurring during the attacks, such as sensorineural hearing loss (SNHL), tinnitus, or aural fullness [1,2]. Most patients progress to chronic imbalance, moderate to severe deafness in the affected ear and, in many cases, persistent and dis-

\footnotetext{
- Received May 14, 2021

Revised August 11, 2021

Accepted August 20, 2021

- Corresponding author: Jose Antonio Lopez-Escamez

Otology and Neurotology Group CTS495, Department of Genomic

Medicine, Centre for Genomics and Oncological Research (GENYO),

Pfizer-University of Granada-Junta de Andalucía, PTS, Granada, Avenida de

la Ilustración, 114, 18016, Granada, Spain

Tel: +34-958-715-500-160

E-mail: antonio.lopezescamez@genyo.es
}

abling tinnitus [3]. The disorder usually begins in one ear with tinnitus and hearing loss (unilateral MD; UMD), but it can evolve to both ears and produce bilateral symptoms (bilateral MD; $\mathrm{BMD}$ ). The diagnostic criteria for MD are based on the joint presentation of vertigo and aural symptoms during the attack, and they were reformulated jointly by the Classification Committee of the Bárány Society, the Japan Society for Equilibrium Research, the European Academy of Otology and Neurotology, the Equilibrium Committee of the American Academy of Otolaryngology-Head and Neck Surgery, and the Korean Balance Society in 2015 [4]. However, these criteria do not consider the clinical heterogeneity observed among patients, and some comorbidities commonly observed in some patients, such as migraine or autoimmune disease (AD), should be considered in the therapeutic management.

\section{CLINICAL SUBGROUPS IN MD}

$\mathrm{MD}$ is not a single disease; instead, it should be considered as a

Copyright $\odot 2022$ by Korean Society of Otorhinolaryngology-Head and Neck Surgery

This is an open-access article distributed under the terms of the Creative Commons Attribution Non-Commercial License (https://creativecommons.org/licenses/by-nc/4.0)

which permits unrestricted non-commercial use, distribution, and reproduction in any medium, provided the original work is properly cited. 

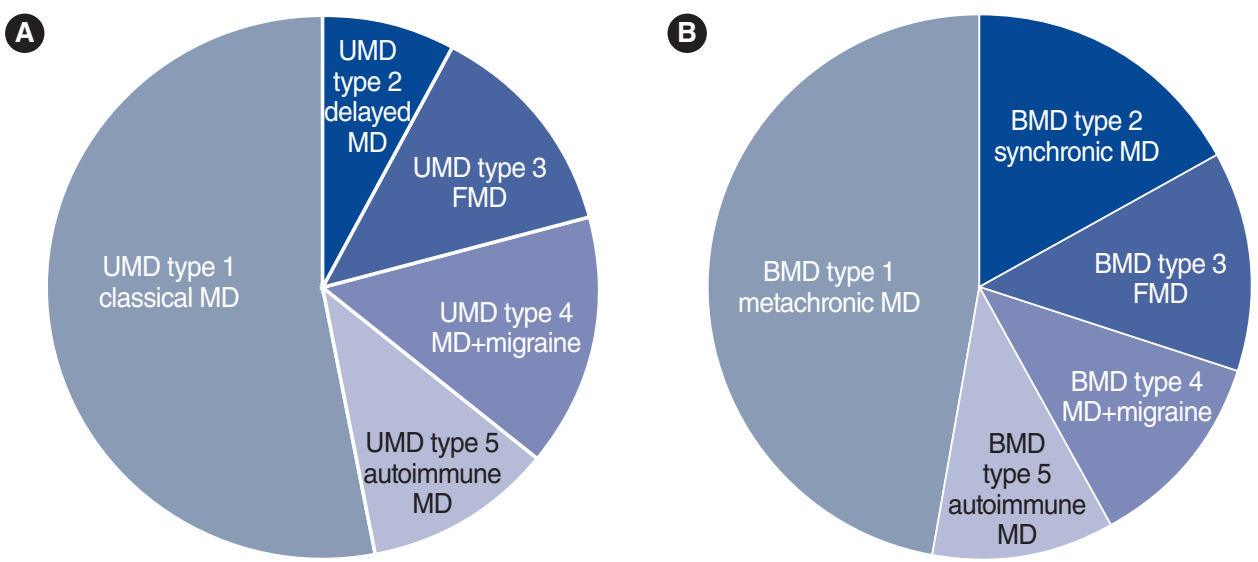

Fig. 1. Clinical subgroups of patients with unilateral Meniere disease (UMD; A) and bilateral Meniere disease (BMD; B). MD, Meniere disease; FMD, familial Meniere disease.

Table 1. Clinical subgroups of patients with UMD and BMD

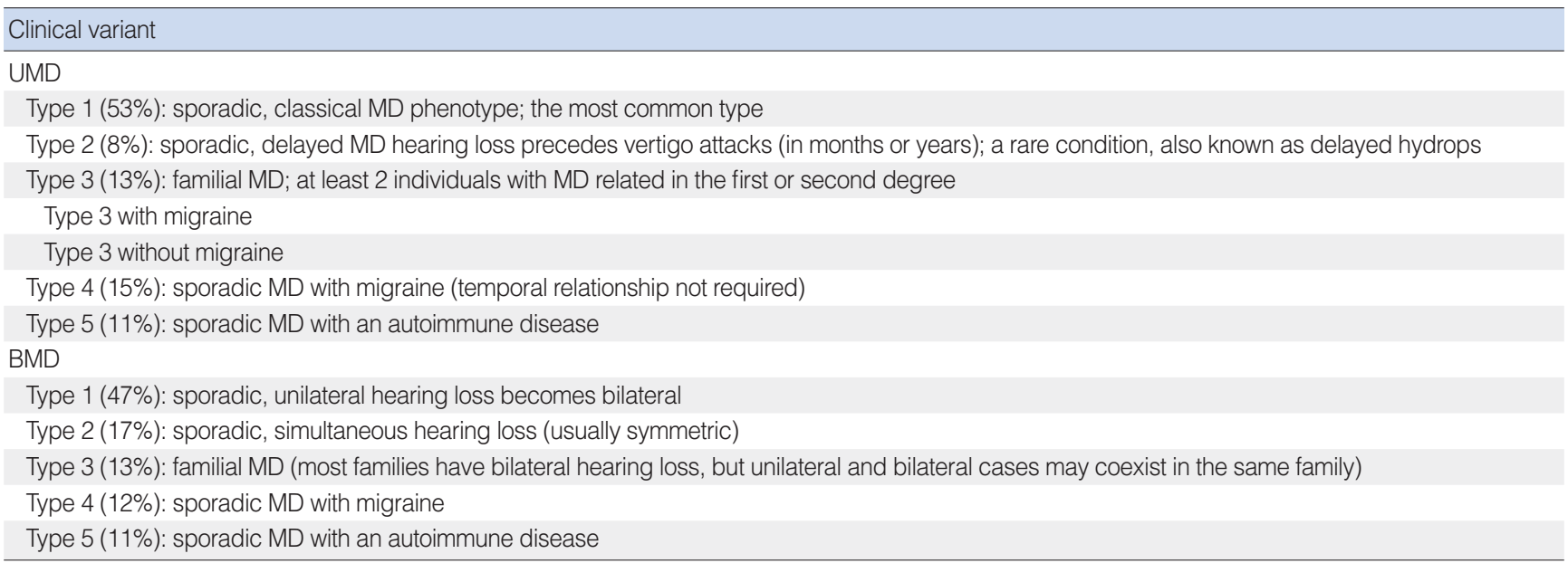

MD, Meniere disease; UMD, unilateral MD; BMD, bilateral MD.

clinical syndrome with different etiologies. Ten clinical subgroups of MD patients have been described using cluster analysis and clinical data from over 1,500 MD patients [5,6], according to four clinical predictors: cochlea-vestibular symptoms occurring after SNHL (delayed hydrops), familial MD (FMD), migraine, and $\mathrm{AD}$. Five subgroups were reported in UMD and five

\section{H I G G H L I I G}

- High levels of interleukin (IL)-1 $\beta$ and tumor necrosis factor- $\alpha$ in some Meniere disease (MD) patients suggest that it is a chronic inflammatory disorder.

- The quantitative trait locus rs4947296 regulates the TWEAK/ FN14 pathway and nuclear factor-kappa B-mediated inflammation in bilateral MD.

- IL-1 $\beta$, CCL3, CCL22, and CXCL1 may be used as biological markers to distinguish $\mathrm{MD}$ and vestibular migraine. were also found in BMD (Fig. 1, Table 1), all with strong potential etiological implications, and six of these subgroups were shared between UMD and BMD (type 3, FMD; type 4, MD with migraine; and type $5, \mathrm{MD}$ with $\mathrm{AD}$ ).

Familial clustering has been reported in about $9 \%$ of cases in southern European populations [7], and in 6\% of cases in South Korea [8], additionally supporting a genetic contribution to the disease [9]. FMD presents an autosomal dominant pattern of inheritance with incomplete penetrance and anticipation, showing an earlier onset compared to sporadic MD [10-12].The families can include patients with UMD or/and BMD; therefore, epigenetic factors might influence unilateral or bilateral involvement [7]. FMD could be split into two subgroups (FMD with and without migraine), confirming the early description of families with MD co-segregating with migraine [13] and the more recent description of FMD without migraine [14,15], as well as reflecting the genetic heterogeneity of FMD. Familial cases may also show recessive inheritance, providing further support that several genes 
contribute to FMD and suggesting that it should be considered a rare polygenic disease [16].

These clinical variants observed in both UMD and BMD validate previous epidemiological studies, but they also indicate a separate role for genetics and autoimmunity as relevant factors influencing the development of MD. While the pathological mechanisms underlying MD remain poorly understood, many studies have postulated that various factors may be involved, including endolymphatic hydrops, allergy, inflammation, infection, and ADs $[17,18]$. Among these theories, it seems that the immune system plays a critical role in a large population of MD patients $[19,20]$. However, the role of the immune system in patients with MD has not been well studied.

\section{THEORY OF IMMUNE INVOLVEMENT INTHE INNER EAR}

The notion that the immune system could have a role in some idiopathic hearing loss and vestibular disorders was introduced during the early 20th century by Joannovic in 1920 [21] and Masugi in 1931 [22]. In 1958, Lehnhardt [23] suspected that certain cases of sudden bilateral hearing loss could be associated with the production of anti-cochlear antibodies. Kikuchi [24] suggested an autoimmune etiology after observing that surgery in one ear affected the other one. Beickert [25] and Yoshihiko and Yukihiro [26] both presented data supporting an autoimmune mechanism in experimental guinea pig cochleae. In 1979, McCabe [27] first described patients with bilateral progressive hearing loss that responded to steroid therapy. The clinical presentation of SNHL can be quite variable, often overlapping with other disorders such as MD or deafness autosomal dominant 9 (DFNA9), which can result in diagnostic confusion. DFNA9 is caused by pathogenic variants in the $\mathrm{COCH}$ gene, which encodes cochlin [28]. Cochlin, a major component of the extracellular matrix in both the cochlea and vestibule of the inner ear, is a potential candidate antigen for autoimmune inner ear disease (AIED) [29] and DFNA9 with or without vestibular abnormalities [30], related to inner ear immunity and stimulation of the secretion of inflammatory cytokines [31]. Hughes et al. [32] reported that over $52 \%$ of patients diagnosed with AIED presented hearing loss and vertigo, suggesting that a continuum might exist between MD and SNHL (Fig. 2).

\section{AUTOIMMUNE MD}

We currently have evidence of inflammation in some inner ear diseases, including MD, progressive SNHL, otosclerosis, and sudden deafness. The prevalence of systemic ADs such as rheumatoid arthritis (RA), ankylosing spondylitis, systemic lupus erythematosus (SLE), and psoriasis in MD patients is 3- to 8-fold

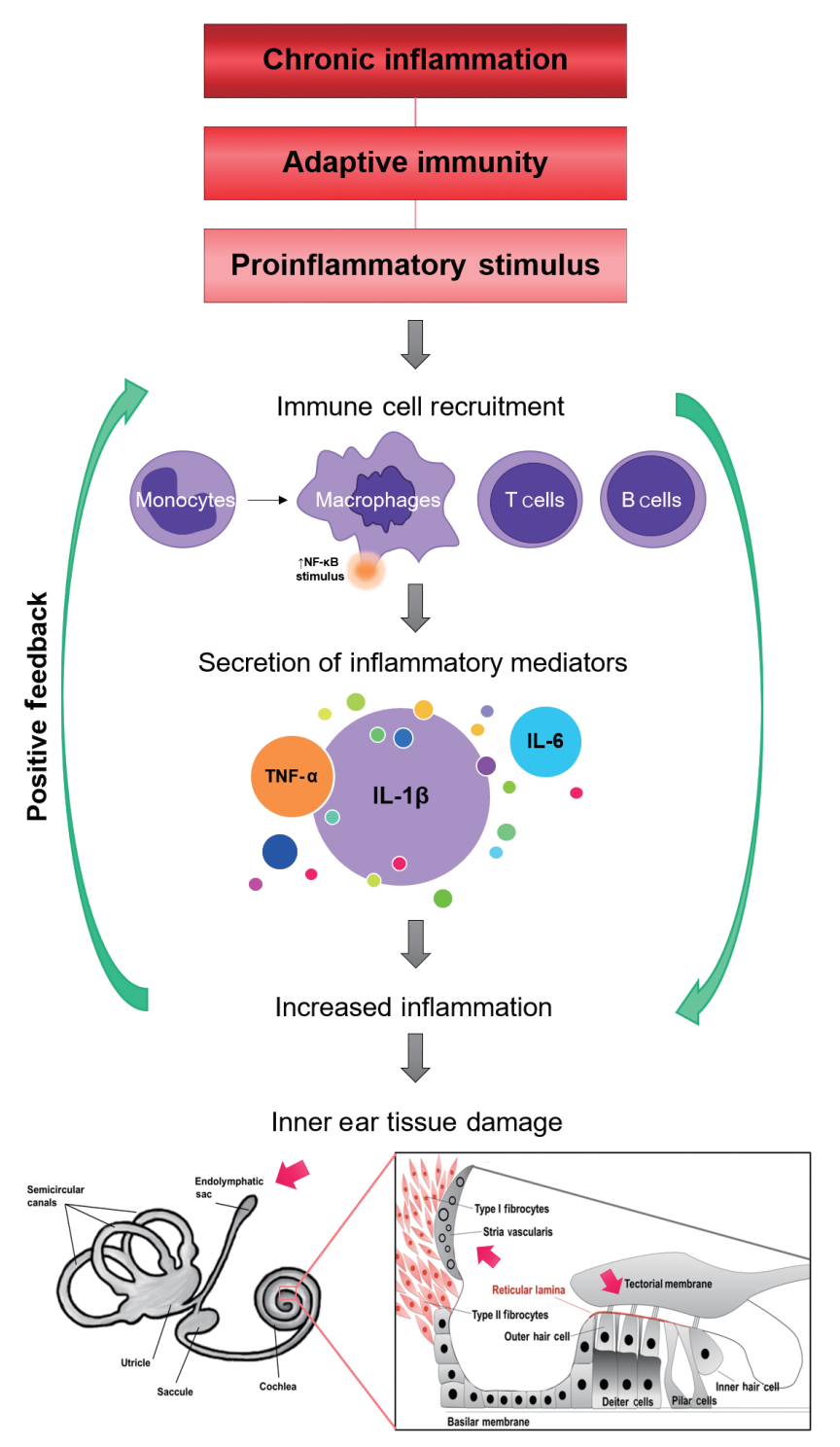

Fig. 2. Potential mechanisms of inflammation in Meniere disease (MD). Red arrows indicate the potential targets most likely to be damaged. NF-kB, nuclear factor-kappa B; TNF, tumor necrosis factor; IL, interleukin.

higher than in the general population [33-35]. According to some studies, autoimmunity seems to be responsible for $6 \%$ of cases of UMD and $16 \%$ of cases of BMD [36]. Based on the findings of proteomic studies performed in small series of patients, autoimmunity has been proposed as a potential cause of MD [20,37]. Approximately one-third of MD cases seem to be of autoimmune origin; however, elevated immune complexes were only found in $7 \%$ of patients with MD [38], and there is no consistent immunological biomarker for the diagnosis of MD. Therefore, several hypotheses have been proposed to explain how inflammation may arise in MD: bystander damage, cross-reactions, intolerance, and genetic factors. These mechanisms are supported by several experimental studies, as detailed below. (1) Antibodies 
or rogue $\mathrm{T}$ cells may cause accidental inner ear damage because the ear shares common antigens with a potentially harmful substance, virus, fungus, or bacterium that the body is battling. This is presently the preferred theory for AIED. (2) The body may not recognize all inner ear antigens. When they are released (perhaps after surgery or an infection), the body may wrongly attack the "foreign" antigens. In the ear, a mechanism that could be involved in so-called sympathetic cochleo-labyrinthitis has been reproduced in animal models [39]. (3) Endolymphatic hydrops can be induced experimentally by injection of antigens or monoclonal antibodies [40]. Inner ear antigens with molecular weights of $28,42,58$, and $68 \mathrm{kDa}$ could be the main components that induce autoimmune MD in guinea pigs [41]. (4) The deposition of circulating immune complexes (CIC) could produce inflammation and interfere with the capability of endolymphatic sac (ES) filtering. Several studies have demonstrated increased CIC levels in $21 \%-96 \%$ of MD patients [42]. (5) Autoantibodies have been found in MD patients' sera [43]. (6) Genetically controlled features of the immune system could increase or otherwise be associated with increased susceptibility to common hearing disorders [44]. Allelic variants in immune response genes related to SNHL progression, such as MICA, TLR10, and NFKB1, have also been reported [45-47]. (7) Frejo et al. [48] have shown that an immune response factor involved in MD is Fn14 (fibroblast growth factor-inducible 14; Tnfrsf12a), the receptor for TWEAK, a multifunctional cytokine (tumor necrosis factor [TNF]-like weak inducer of apoptosis; Tnfsf12), which is a member of the TNF superfamily. The TWEAK/Fn14 pathway is involved in the modulation of inflammation in several chronic ADs, including multiple sclerosis (MS), SLE, RA, and ulcerative colitis [49]. It has been discovered that one of its allelic variants (rs4947296) is a quantitative trait locus that regulates the expression of multiple genes in the TWEAK/Fn14 pathway in peripheral blood mononuclear cells. This locus leads to an inflammatory response mediated by the transcription factor nuclear factor-kappa B (NF- $\mathrm{B}$ ) in MD. This variant was also associated with bilateral MD in the Spanish population, and it appears in up to $18 \%$ of patients with comorbid autoimmune conditions.

However, the evidence supporting the hypothesis of autoimmunity is limited and the involved immunological mechanisms remain unclear. Autoimmune MD should be diagnosed if a patient fulfills the diagnostic criteria for MD and one of the following ADs: autoimmune thyroid disease, psoriasis, autoimmune arthritis, ankylosing spondylitis, or SLE. All of these conditions have been previously associated with MD in epidemiological studies in European populations [33,35].

\section{CYTOKINES INTHE INNER EAR}

The existence of immunological activity in the anterior labyrinth has been widely described, both in humans and animal models.
Immune responsiveness in the inner ear was initially associated with the ES [50-52] since it possesses immunological capacities and is responsible for a major part of the trans-epithelial ion transport occurring within the inner ear [53]. However, the presence of immune capacity in the cochlea has since been established [54] through the recent demonstration of IBA1-expressing macrophages $[55,56]$ in the human ES and cochlea that express major histocompatibility complex type II (MHCII). Kampfe Nordstrom et al. [57] identified that the macrophage population of the stria vascularis, spiral ligament, and spiral ganglion expressed MHCII, which is crucial for initiating antigen-specific immune responses. Furthermore, it was proposed that there could be uptake and processing of antigens from the ES lumen, due to the co-expression of IBA1 and MHCII in epithelial cells and transepithelial migration. Additionally, Moller et al. [58] found gene expression for both the cellular and humoral innate immunesystem, including toll-like receptors 4 and 7, beta-defensin, and lactoferrin in the ES. These findings provide molecular evidence of an immunological capacity of the ES to recognize and process antigens for immune responses.

Autoinflammatory diseases are triggered by an overactive inflammatory response leading to immune dysregulation, fundamentally mediated by interleukin (IL)-1 $\beta$, type I interferon (IFN)mediated responses, and the transcription factor NF- $\kappa B$ [59]. These diseases normally manifest in the perinatal period; however, late-onset forms are diagnosed in adulthood [60]. Some of these disorders have symptoms that mimic allergic and immunodeficiency disorders. Additionally, many of them are accompanied by SNHL as a symptom, suggesting that potentially similar hearing loss pathogenesis molecular mechanisms may exist. Over the last decade, new molecules have been explored to detect the role of cytokines in $\mathrm{AD}$, and new drugs are being developed to interfere with them. Around $60 \%$ of MD patients have antibodies in their sera against proteins in the inner ear, and there is evidence of the presence of cytokines in the cochlea, including IL-1 $\alpha$, TNF- $\alpha$, NF-k $\beta$ P65, P50, and Ik $\beta \alpha$ [61].

\section{TNF- $\alpha$}

TNF- $\alpha$ is a cytokine that binds to TNFRSF1A/TNFR1 and TNFRSF1B/TNFBR. It is mainly secreted by macrophages, induces the infiltration of immunocompetent cells into the tissues, and amplifies the immune response. It is a potent pyrogen causing fever by direct action or by stimulation of IL-1 secretion, and under certain conditions it can stimulate cell proliferation and induce cell differentiation. It may also impair regulatory T-cell (Treg) function in individuals with RA via FOXP3 dephosphorylation. TNF- $\alpha$ upregulates the expression of protein phosphatase 1, which dephosphorylates the key Ser418 residue of FOXP3, thereby inactivating FOXP3 and rendering Treg cells functionally defective [62].

In a small study population of 15 subjects, Ren et al. [63] reported that both TNF- $\alpha$ and IL- 6 levels were significantly elevat- 
ed in patients with sudden SNHL and progressive SNHL when compared to controls. This study was followed by a preliminary report on the use of etanercept [64], a well-known TNF- $\alpha$ blocker used in ADs such as psoriasis and RA, for patients with immune-mediated cochleovestibular disorders who did not respond to conventional therapies. They reported a $92 \%$ success rate in hearing loss improvement with etanercept therapy.

Animal models of labyrinthitis have been used to study the role of TNF- $\alpha$ in recruiting inflammatory cells to the cochlea and its role in resultant hearing loss [65]. While some researchers reported that, in an animal model, etanercept had a protective effect on hearing loss [66], others found it to be less effective than prednisone, with a greater potential for adverse effects [67]. In contrast, human studies performed in patients with sudden SNHL showed increased levels of TNF- $\alpha$ [68], while other studies presented opposite results [69]. In 2012, Svrakic et al. [70], performed a prospective study in patients with immune-mediated SNHL (IM-SNHL) where they concluded that TNF- $\alpha$ had the potential for use as both a diagnostic marker for IM-SNHL and a prognostic biomarker for corticosteroid response in the disease. Three years later, Pathak et al. [71] demonstrated the TNF- $\alpha$ could be attenuated in a subset of patients with AIED using N-acetylcysteine (NAC) [72], which might serve as a beneficial adjunctive therapy in hearing restoration. NAC has been shown to have a protective adjunct role in idiopathic sudden hearing loss, as the addition of NAC to corticosteroid therapy resulted in better hearing recovery than with corticosteroids alone [73].

\section{IL-1 $\beta$}

IL- $1 \beta$ is produced by monocytes as an inactive $31 \mathrm{kDa}$ precursor, termed pro-IL-1 $\beta$, in response to molecular motifs carried by pathogens referred to as pathogen-associated molecular patterns (PAMPs). Pro-IL-1 $\beta$ is cleaved by the protease caspase-1 [74]. The activation of caspase- 1 occurs via recruitment to a multi-protein complex termed the inflammasome [75], which leads to an active $17-\mathrm{kDa}$ form of IL-1 [76].

Over a decade ago, the critical role of the IL-1 family as regulators of inflammation and immunity became evident [77]. Early immune system reactions to PAMPs may drive many of the later adaptive T cell responses that maintain the disease. IL- $1 \beta$ is a key proinflammatory cytokine involved in the progression of many autoinflammatory diseases and ADs, including AIED and MD [78]. During the immune response, the absence of the expression of IL-1 receptor antagonist (IL-1Ra) or other molecules that oppose the IL-1 $\beta$ inflammatory cascade could promote the development of ADs and autoinflammatory diseases. IL-1 $\beta$ is crucial in the development of inflammation and the aggravation of many autoinflammatory diseases and ADs. IL- $1 \beta$ plays a homeostatic role in routine biological processes, but its constitutive overproduction is associated with chronic diseases such as RA, osteoarthritis, type 1 diabetes, MS, inflammatory bowel disease, Muckle-Wells syndrome, DFNA34, and corticosteroid-resistant
AIED [79-87]. IL-1 $\beta$ is processed by the inflammasome, and genetic mutations occurring in its different components and the resultant overproduction of IL-1 $\beta[88,89]$ are clinically manifest in many autoinflammatory diseases [90-92]. The role of IL-1 $\beta$ in hearing disorders is essentially unknown; nonetheless, there are examples of its involvement in both animal models of AIED and clinical autoinflammatory disorders with associated SNHL [93]. SNHL has been observed as a component of clinical diseases involving IL-1 $\beta$ dysregulation, such as neonatal-onset multisystemic inflammatory disease syndrome [94] and Muckle-Wells syndrome [95]. Likewise, improvement of SNHL has been observed in response to treatment with anakinra, a soluble IL-1Ra, in Muckle-Wells syndrome and AIED [96,97]. A recent report found that the processed $28-\mathrm{kDa}$ form of IL-1 $\beta$, which is uniquely generated by caspase-7, in patients with AIED can activate further downstream proinflammatory cytokines [78].

\section{CYTOKINES AND MD}

Frejo et al. [98] observed that basal levels of the proinflammatory cytokines TNF- $\alpha$, IL-1 $\beta$, and IL- 6 could be increased in some MD patients. This observation was found in both UMD and BMD patients, as well as in healthy individuals after stimulation with lipopolysaccharide (LPS), which was used as positive control for inflammation (Fig. 3). They observed two subgroups of MD patients according to their IL-1 $\beta$ profile (patients with high levels of IL-1 $\beta$ and patients with low levels of IL-1 $1 \beta$ ), and these groups could have different immune responses or functional states of the immune system [98]. Later, Flook et al. [99], showed that patients with MD or vestibular migraine had separate pro-inflammatory profiles. A cytokine panel including IL$1 \beta$, CCL3, CCL22, and CXCL1 could be used as biological markers for the differential diagnosis of vestibular migraine and MD. The expression of IL-1 $\beta$, CCL3, CCL22, and CXCL1 has been previously observed in mouse cochlear tissue [100], and

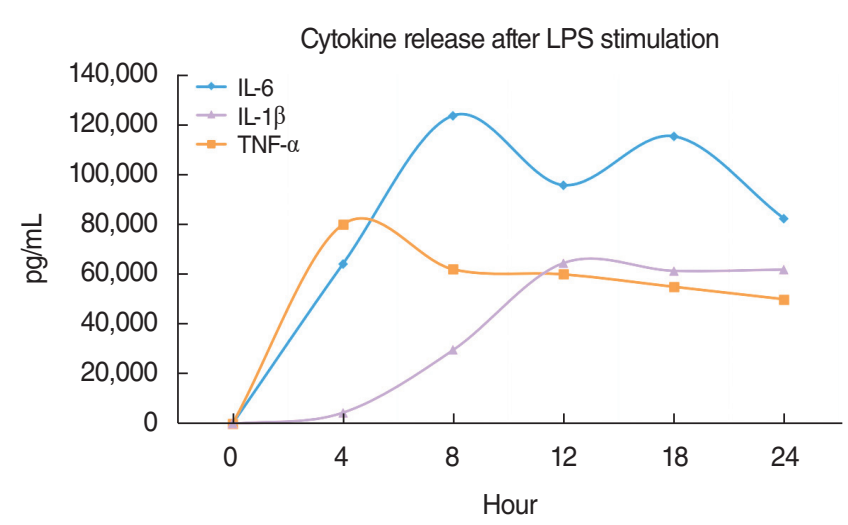

Fig. 3. Temporal profile of cytokine release after lipopolysaccharide (LPS) stimulation in peripheral blood mononuclear cells from a healthy individual. IL, interleukin; TNF, tumor necrosis factor. 
IL-1 $\beta$, CCL3, and CXCL1 have also been found in cells from the ES [101]. Interestingly, CXCL1 is the only chemokine that has been found to be expressed in mouse vestibular cells [102], and it showed the highest expression of the four cytokines in all tissues. Therefore, cytokine and chemokine profiles could be used to define the immune response and the functional state of the immune response in $\mathrm{MD}$.

\section{POTENTIAL DRUGSTHAT COULD REGULATE THE IMMUNE RESPONSE IN MD}

The treatment of MD primarily focuses either on lowering endolymphatic pressure or controlling its symptoms during vertigo episodes. No treatment cures the disease; therefore, treatment involves a trade-off of improving symptoms with the fewest side effects. Consequently, treatment starts with the most conventional modalities: diet, lifestyle changes to reduce stress, and medications to control vertigo. Hence, better treatment options are needed. Implicitly, all clinically employed drugs have effects on biological systems other than those for which they were designed. This characteristic of drugs could result in a positive outcome, as occurs when a drug established for one therapeutic indication finds utility for another indication (known as "repurposing"). For example, steroids, which are commonly used in ADs, can benefit patients with sudden hearing loss or autoimmune MD (Table 2).

Several actors including immune and non-immune cells, cytokines such as TNF- $\alpha$, IL-1 $\beta$, IL-6, or type I IFN, and transcription factor NF-kB play central roles in inflammation. Synergistic in-

Table 2. Potential beneficial drugs for the treatment of autoimmune/ autoinflammatory Meniere disease

\begin{tabular}{lll}
\hline Target & \multicolumn{1}{c}{ Drug } & \multicolumn{1}{c}{ Function } \\
\hline TWEAK & BIIB023 & Inhibits binding between TWEAK and Fn14 \\
NF-KB & Cepharanthine & NFKB p105 inhibitor \\
& Resveratrol & NFKB p65 and IkB inhibitor \\
IL-6 & Siltuximab & Neutralizes IL-6 binding to its receptors \\
& Sarilumab & Binds to both sIL-6R and mIL-6R \\
IL-6R & Tocilizumab & Inhibits binding between IL-6 and IL-6R \\
& Satralizumab & Inhibits binding between IL-6 and IL-6R \\
TNF & Etanercept & Binds to TNF and inhibits its binding with TNF-R \\
& Infliximab & Neutralizes the activity of TNF- $\alpha$ \\
& Adalimumab & TNF inhibitor \\
& Certolizumab & Neutralizes TNF- $\alpha$ dose dependently \\
& Golimumab & Inhibits both solTNF- $\alpha$ and tmTNF- $\alpha$ \\
IL-1 $\beta$ & Rilonacept & IL-1 $\alpha / \beta$ blocker \\
& Anakinra & Recombinant IL-1Ra, IL-1 $\beta$ inhibitor \\
& Celastrol & Anti-inflammatory agent \\
& Canakinumab & Antibody anti-IL-1 $\beta$ \\
\hline
\end{tabular}

NF-kB, nuclear factor-kappa B; IL, interleukin; IL-6R, IL-6 receptor; TNF, tumor necrosis factor; sIL-6R, soluble IL-6R; mIL-6R, membrane IL-6R; IL$1 \mathrm{Ra}, \mathrm{IL}-1$ receptor antagonist. teractions between NF- $\mathrm{KB}$ and other transcription factors induce the hyper-activation of NF- $\mathrm{kB}$, followed by the production of various inflammatory cytokines [103]. TWEAK is a multifunctional cytokine that regulates several cellular responses, including inflammation, cellular adhesion, proliferation, and apoptosis $[104,105]$. TWEAK is expressed in several cell types, including monocytes/macrophages, dendritic cells, activated T cells, astrocytes and microglia, endothelial cells, and erythroblasts. The biological activity of TWEAK is mediated through its receptor, fibroblast growth factor-inducible 14 (Fn14), which is highly expressed in epithelial cells and induced in several human diseases [106]. Increased levels of TWEAK and/or Fn14 have also been found to be associated with the pathogenesis of RA [107], SLE [108], MS [109], or neuroinflammation [110]. The binding of TWEAK to Fn14 induces both acute activation of the canonical NF- $\mathrm{kB}$ pathway and prolonged activation of the non-canonical NF- $\kappa B$ pathway [104]. Furthermore, the non-canonical NF- $\mathrm{kB}$ pathway plays a key role in immunity and immune-mediated disorders such as SLE. The non-canonical NF- $\mathrm{kB}$ pathway relies on the phosphorylation-induced p100 processing, which is triggered by signaling from a subset of TNFR members, including Fn14, TNFR2, BAFFR, CD40, LTßR, and RANK [111]. Most of these signals are regulatory elements of the immune response, supporting the hypothesis that the allelic variants of immune response genes can modify the clinical course in MD. Inflammation may occur in the ES, since proteomic studies have found high contents of immunoglobulins (Igs) in the ES [20]. Endolymph and perilymph homeostasis is maintained at the cochlea and vestibular semicircular canals at multiple sites, including the spiral ligament, the stria vascularis, the cochlear and vestibular non-sensory epithelial cells, and the ES. The sac is a small organ located in the posterior cranial fossa and plays a crucial role both in the maintenance of endolymph composition and in the innate immune response [58]. We hypothesize that, after exposure to an environmental trigger, the carriers of the risk haplotype could have an abnormal NF-kB-mediated inflammatory response in the ES, causing an ionic imbalance in the endolymph, leading to the accumulation of endolymph at the cochlear duct. Therefore, the TWEAK/Fn14 and NF-kB pathways have potential druggable targets.

Although there are no approved drugs for either TWEAK or its receptor Fn14, BIIB023 is an anti-TWEAK monoclonal antibody that demonstrated a favorable safety and tolerability profile in a phase 1 study of RA patients. In contrast, regulation of $\mathrm{NF}-\mathrm{kB}$ pathways play a pivotal role in cellular responses to changes in the environment. At present, several biologics and molecules that modulate both the canonical and non-canonical pathways are in the market or in clinical trials. Moreover, many marketed drugs that were later observed to also have NF-kB targeting activity were repurposed for new therapeutic interventions [112]. There are over 700 known inhibitors of NF-kB signaling at several levels [113] that could be potential druggable targets. 
The NF-kB pathway in general can be regulated at three different points: (1) blocking the signal reception by interfering with the receptor-ligand binding, thereby abolishing the signaling cascade at the initial stage; (2) interfering with any of the factors involved in the cascade in the cytoplasm; and (3) targeting the nuclear translocation of NF-kB factors or interfering with their DNA binding.

Cepharanthine (CEP) has a multifactorial mechanism of action. The drug exerts membrane effects such as the modulation of efflux pumps and membrane rigidification, as well as various intracellular and nuclear effects. CEP interferes with numerous metabolic axes, primarily with the AMP-activated protein kinase (AMPK) and NF-kB signaling pathways. Specifically, its anti-inflammatory effects rely on AMPK activation and NF-kB inhibition. Resveratrol inhibits NF-kB signaling through suppression of p65 and IкB kinase activities [114].

Several cytokines could also be potential targets to treat MD since they mediate an overactive inflammatory response that triggers immune dysregulation in inflammatory diseases. Type I IFN can have dual and opposing roles in immunity, with effects that can be beneficial or detrimental to the individual depending on whether IFN pathway activation is temporary or persistent. When dysregulated, the type I IFN system drives many inflammatory diseases, including SLE, myositis, RA, and Sjogren syndrome [115]. IL-6 is a pleiotropic pro-inflammatory cytokine produced by a variety of cell types, including monocytes, lymphocytes T- and B-cells, and fibroblasts. IL-6 has been shown to be involved in diverse physiological processes in response to infections and tissue damage, and it contributes to host defense through the stimulation of acute-phase responses, hematopoiesis, and immune reactions. While its expression is firmly regulated by transcriptional and posttranscriptional mechanisms, dysregulated continual synthesis of IL-6 exerts a pathological effect on chronic inflammation and autoimmunity [116].

There are several approved drugs against IL-6 and its receptors (IL-6R and IL-6ST). Siltuximab is a recombinant human-mouse chimeric monoclonal antibody that binds to IL-6. It prevents the binding of IL- 6 to both soluble and membrane-bound IL-6 receptors (soluble IL-6R [sIL-6R] and membrane IL-6R [mIL-6R]), inhibiting IL-6 signaling. Sarilumab is a human recombinant monoclonal antibody of the IgG1 subclass. It binds to both sIL6R and mIL-6R, and it has been shown to inhibit IL-6-mediated signaling through these receptors. Tocilizumab is a first-generation antibody directed against IL-6R. It inhibits binding between IL-6 and IL-6R. Satralizumab is a second-generation anti-IL-6R monoclonal antibody designed to have enhanced antigen-neutralizing capacity and a longer plasma half-life than tocilizumab. Furthermore, several anti-IL6 agents, such as clazakizumab, and anti-IL6R agents, such as olokizumab and vobarilizumab, are also being studied.

TNF has two forms: soluble (solTNF) and transmembrane (tmTNF). Although both forms are biologically active, they have clearly different roles. tmTNF has been shown to play a crucial role in maintaining the physiological innate immune response to infections [117], while the main role of solTNF is to drive the inflammatory response. This function has been proven in animal studies showing that inhibition of solTNF causes an anti-inflammatory effect. Inhibition of tmTNF, in contrast, results in increased sensitivity to infection and exacerbation of demyelination. Five TNF inhibitors have been approved for the treatment of various inflammatory diseases: etanercept, infliximab, adalimumab, certolizumab, and golimumab. All of these agents competitively inhibit the binding of TNF to its receptors. Nevertheless, they differ in both pharmacokinetic and pharmacodynamic characteristics, leading to significant differences in their clinical efficacy and indications. Alongside the approved therapies (RA, ankylosing spondylitis, Crohn disease, and psoriasis), TNF inhibitors are also repurposed for off-label indications such as SLE, scleroderma, Sweet syndrome, or Sjogren syndrome, even though in most of these cases large, controlled studies are still lacking [118].

Adalimumab is a humanized monoclonal antibody that, on one hand, specifically binds to TNF and blocks its interaction with endogenous TNF receptors to modulate inflammation, while on the other hand, it induces apoptosis of lymphocytes that are abnormally activated. Certolizumab is a polyethylene-glycolated Fab' fragment of TNF antibody that specifically binds to TNF- $\alpha$ and neutralizes it in a dose-dependent manner. It also inhibits the production of LPS-induced TNF- $\alpha$ and IL-1 $1 \beta$. Golimumab is a human monoclonal antibody with immunosuppressive features that targets both solTNF- $\alpha$ and tmTNF- $\alpha$. The TNF-golimumab complex neutralizes the expression of C-reactive protein levels, IL-6, intercellular adhesion molecule-1, matrix metalloproteinase-3, and vascular endothelial growth factor induced by TNF- $\alpha$, demonstrating that golimumab is an effective modulator of inflammatory markers [119]. Etanercept is a recombinant version of soluble human TNF receptor fused to an IgG Fc fragment that binds specifically to TNF and inhibits its binding with endogenous TNF receptors. The products of infliximab neutralize the biological activity of TNF- $\alpha$ by binding with high affinity to both solTNF- $\alpha$ and tmTNF- $\alpha$, inhibiting their binding with TNF- $\alpha$ receptors. Infliximab products do not neutralize TNF- $\beta$ (lymphotoxin-alpha), a related cytokine that employs the same receptors as TNF- $\alpha$.

More than any other cytokine family, the IL- 1 family is primarily associated with innate immunity, and even though the inflammatory characteristics of the IL- 1 family dominate in the innate immune response, they can also play a role in acquired immunity [120]. IL-1 $\beta$ is a proinflammatory cytokine that exerts pleiotropic effects on a variety of cells and plays key roles in acute and chronic inflammatory disorders and ADs. Its overproduction is implicated in the pathophysiological changes that arise during different disease states, such as RA, MS, Alzheimer disease, neuropathic pain, and inflammatory bowel disease. The proinflammatory activities of IL-1 are controlled by various en- 
dogenous inhibitors, such as IL-1Ras and membrane-bound and soluble IL-1 receptor type II and IL-1 receptor accessory proteins $[121,122]$.

Canakinumab is a human IgGк monoclonal antibody targeting IL-1 $\beta$ [123]. Rilonacept is a dimeric, glycosylated fusion protein comprising the extracellular domain of IL-1R and the Fc domain of human IgG1. Similar to canakinumab, rilonacept is intended to bind and neutralize IL-1. It binds to both IL-1 $\alpha$ and IL-1 $\beta$ with high affinity, and so it has been suggested that rilonacept might have better inhibitory effects in vivo than other IL-1 blockers [123]. Anakinra is a recombinant IL-1Ra that has been shown to be effective for the treatment of a subgroup of AIED patients [97]. Potentially, celastrol, which has several cellular targets-interfering with the production of cytokines, chemokines, and inflammatory mediators such as IL- $1 \beta$ and TNF- $\alpha$; inhibiting cell invasion and proliferation; and suppressing bone resorption - could constitute a potential candidate for the treatment of inflammatory diseases.

\section{CONCLUSION}

All these approved drugs could potentially be beneficial for the treatment of autoimmune/autoinflammatory MD. Since no cure exists for MD, preclinical research and clinical trials repurposing these drugs should be promoted in the next few years. We propose, for instance, to start employing these drugs as secondary treatments. Clinical trials have demonstrated the efficacy of tocilizumab, a humanized anti-IL-6 receptor antibody, for patients with RA and Castleman disease [116], and it may play a role in asthma and other inflammatory pulmonary diseases [124], leading to approval of this innovative drug for the treatment of these diseases. Since IL-6 has been demonstrated to play a significant role in the development of various autoimmune and inflammatory diseases, recruiting MD patients with a history of any of these comorbidities could be a potential starting point to treat them.

\section{CONFLICT OF INTEREST}

No potential conflict of interest relevant to this article was reported.

\section{ACKNOWLEDGMENTS}

We acknowledge all patients and participants in the Meniere Disease Consortium (MeDiC). Jose Antonio Lopez-Escamez has received funds to support research on the immune response in Meniere disease from Instituto de Salud Carlos III and European Regional Funds (grants no. PI17/1644 and PI20-1126) and the
Andalusian Health Department (grants no. PE-0356-2018 and PI027-2020).

\section{ORCID}

Lidia Frejo https://orcid.org/0000-0002-6104-0941 Jose Antonio Lopez-Escamez

https://orcid.org/0000-0002-8583-1430

\section{AUTHOR CONTRIBUTIONS}

Conceptualization: all authors. Data curation: LF. Formal analysis: all authors. Funding acquisition: JALE. Methodology: LF. Project administration: JALE. Visualization: all authors. Writingoriginal draft: LF.Writing-review \& editing: all authors.

\section{REFERENCES}

1. Amanat S, Requena T, Lopez-Escamez JA. A systematic review of extreme phenotype strategies to search for rare variants in genetic studies of complex disorders. Genes (Basel). 2020 Aug;11(9):987.

2. Espinosa-Sanchez JM, Lopez-Escamez JA. Meniere's disease. Handb Clin Neurol. 2016;137:257-77.

3. Stahle J, Friberg U, Svedberg A. Long-term progression of Meniere's disease. Acta Otolaryngol Suppl. 1991;485:78-83.

4. Lopez-Escamez JA, Carey J, Chung WH, Goebel JA, Magnusson M, Mandala M, et al. Diagnostic criteria for Meniere's disease. JVestib Res. 2015 Mar;25(1):1-7.

5. Frejo L, Soto-Varela A, Santos-Perez S, Aran I, Batuecas-Caletrio A, Perez-GuillenV, et al. Clinical subgroups in bilateral Meniere disease. Front Neurol. 2016 Oct;7:182.

6. Frejo L, Martin-Sanz E, Teggi R, Trinidad G, Soto-Varela A, SantosPerez S, et al. Extended phenotype and clinical subgroups in unilateral Meniere disease: a cross-sectional study with cluster analysis. Clin Otolaryngol. 2017 Dec;42(6):1172-80.

7. Requena T, Espinosa-Sanchez JM, Cabrera S, Trinidad G, Soto-Varela A, Santos-Perez S, et al. Familial clustering and genetic heterogeneity in Meniere's disease. Clin Genet. 2014 Mar;85(3):245-52.

8. Lee JM, Kim MJ, Jung J, Kim HJ, Seo YJ, Kim SH. Genetic aspects and clinical characteristics of familial Meniere's disease in a South Korean population. Laryngoscope. 2015 Sep;125(9):2175-80.

9. Roman-Naranjo P, Gallego-Martinez A, Lopez Escamez JA. Genetics of vestibular syndromes. Curr Opin Neurol. 2018 Feb;31(1): 105-10.

10. Birgerson L, Gustavson KH, Stahle J. Familial Meniere's disease: a genetic investigation. Am J Otol. 1987 Jul;8(4):323-6.

11. Klar J, Frykholm C, Friberg U, Dahl N. A Meniere's disease gene linked to chromosome 12p12.3. Am J Med Genet B Neuropsychiatr Genet. 2006 Jul;141(5):463-7.

12. Morrison AW, Bailey ME, Morrison GA. Familial Meniere's disease: clinical and genetic aspects. J Laryngol Otol. 2009 Jan;123(1):29-37.

13. Oliveira CA, Ferrari I, Messias CI. Occurrence of familial Meniere's syndrome and migraine in Brasilia.Ann Otol Rhinol Laryngol. 2002 Mar;111(3 Pt 1):229-36.

14. Arweiler-Harbeck D, Horsthemke B, Jahnke K, Hennies HC. Genetic aspects of familial Meniere's disease. Otol Neurotol. 2011 Jun; 
32(4):695-700.

15. Hietikko E, Kotimaki J, Okuloff A, Sorri M, Mannikko M. A replication study on proposed candidate genes in Meniere's disease, and a review of the current status of genetic studies. Int J Audiol. 2012 Nov;51(11):841-5.

16. Roman-Naranjo P, Gallego-Martinez A, Soto-Varela A, Aran I, Moleon M, Espinosa-Sanchez JM, et al. Burden of rare variants in the OTOG gene in familial Meniere's disease. Ear Hear. 2020 Nov/Dec; 41(6):1598-605.

17. Sajjadi H, Paparella MM. Meniere's disease. Lancet. 2008 Aug; 372(9636):406-14.

18. Paparella MM, Djalilian HR. Etiology, pathophysiology of symptoms, and pathogenesis of Meniere's disease. Otolaryngol Clin North Am. 2002 Jun;35(3):529-45.

19. Greco A, Gallo A, Fusconi M, Marinelli C, Macri GF, de Vincentiis M. Meniere's disease might be an autoimmune condition? Autoimmun Rev. 2012 Aug;11(10):731-8.

20. Kim SH, Kim JY, Lee HJ, Gi M, Kim BG, Choi JY.Autoimmunity as a candidate for the etiopathogenesis of Meniere's disease: detection of autoimmune reactions and diagnostic biomarker candidate. PLoS One. 2014 Oct;9(10):e111039.

21. Joannovic D. ZurWirkung fermentativ gewonner spaltungsprodukte aus Geweben und Bakterien. Wein KlinWschr. 1920;70:1410-11.

22. Masugi M,Tomizuka Y. Uber die spezifisch zytotoxischen Veranderungen der Niere und sehr Leber durch das spezifische Antiserum (Nephrotoxin und Hepatoxin).Trans Jap Pathol. 1931;21:329-41.

23. Lehnhardt E. Pathogenesis of sudden hearing disorders. Dtsch Gesundheitsw. 1958 Jan;13(4):105-9.

24. Kikuchi M. On the "sympathetic otitis." Zibi Rinsyo Kyoto. 1959; 52:600.

25. Beickert P. On the problem of perception deafness and autoallergy. Z Laryngol Rhinol Otol. 1961 Nov;40:837-42.

26. YoshihikoT, Yukihiro S. Studies on experimental allergic (isoimmune) labyrinthitis in guinea pigs. Acta Otolaryngol. $1964 \mathrm{Jul} ; 58: 49-64$.

27. McCabe BF. Autoimmune sensorineural hearing loss. Ann Otol Rhinol Laryngol. 1979 Sep-Oct;88(5 Pt 1):585-9.

28. Verdoodt D, Van Camp G, Ponsaerts P,Van RompaeyV. On the pathophysiology of DFNA9: effect of pathogenic variants in the $\mathrm{COCH}$ gene on inner ear functioning in human and transgenic mice. Hear Res. 2021 Mar;401:108162.

29. Baruah P. Cochlin in autoimmune inner ear disease: is the search for an inner ear autoantigen over? Auris Nasus Larynx. 2014 Dec; 41(6):499-501.

30. Danial-Farran N, Chervinsky E, Nadar-Ponniah PT, Cohen Barak E, Taiber S, Khayat M, et al. Homozygote loss-of-function variants in the human $\mathrm{COCH}$ gene underlie hearing loss. Eur J Hum Genet. $2021 \mathrm{Feb} ; 29(2): 338-42$.

31. Jung J, Yoo JE, Choe YH, Park SC, Lee HJ, Lee HJ, et al. Cleaved cochlin sequesters pseudomonas aeruginosa and activates innate immunity in the inner ear. Cell Host Microbe. 2019 Apr;25(4): 513-25.

32. Hughes GB, Kinney SE, Barna BP, Calabrese LH. Autoimmune reactivity in Meniere's disease: a preliminary report. Laryngoscope. 1983 Apr;93(4):410-7.

33. Tyrrell JS, Whinney DJ, Ukoumunne OC, Fleming LE, Osborne NJ. Prevalence, associated factors, and comorbid conditions for Meniere's disease. Ear Hear. 2014 Jul-Aug;35(4):e162-9.

34. Hietikko E, Kotimaki J, Sorri M, Mannikko M. High incidence of Meniere-like symptoms in relatives of Meniere patients in the areas of Oulu University Hospital and Kainuu Central Hospital in Finland. Eur J Med Genet. 2013 Jun;56(6):279-85.

35. Gazquez I, Soto-Varela A, Aran I, Santos S, Batuecas A, Trinidad G, et al. High prevalence of systemic autoimmune diseases in patients with Meniere's disease. PLoS One. 2011;6(10):e26759.
36. Bovo R, Ciorba A, Martini A. Vertigo and autoimmunity. Eur Arch Otorhinolaryngol. 2010 Jan;267(1):13-9.

37. Chiarella G, Di Domenico M, Petrolo C, Saccomanno M, Rothenberger R, Giordano A, et al.A proteomics-driven assay defines specific plasma protein signatures in different stages of Meniere's disease. J Cell Biochem. 2014 Jun;115(6):1097-100.

38. Lopez-Escamez JA, Saenz-Lopez P, Gazquez I, Moreno A, Gonzalez-Oller C, Soto-Varela A, et al. Polymorphisms of CD16A and CD32 Fc $\gamma$ receptors and circulating immune complexes in Meniere's disease: a case-control study. BMC Med Genet. 2011 Jan;12:2.

39. Gloddek B,ArnoldW. Clinical and experimental studies of autoimmune inner ear disease. Acta Otolaryngol Suppl. 2002;(548):10-4.

40. Yoo TJ, Yazawa Y, Tomoda K, Floyd R. Type II collagen-induced autoimmune endolymphatic hydrops in guinea pig. Science. 1983 Oct; 222(4619):65-7.

41. Yoo TJ. Etiopathogenesis of Meniere's disease: a hypothesis. Ann Otol Rhinol Laryngol Suppl. 1984 Sep-Oct;113:6-12.

42. Brookes GB. Circulating immune complexes in Meniere's disease. Arch Otolaryngol Head Neck Surg. 1986 May;112(5):536-40.

43. Yoo TJ, Shea J Jr, Ge X, Kwon SS, Yazawa Y, Sener O, et al. Presence of autoantibodies in the sera of Meniere's disease. Ann Otol Rhinol Laryngol. 2001 May;110(5 Pt 1):425-9.

44. Bernstein JM, Shanahan TC, Schaffer FM. Further observations on the role of the MHC genes and certain hearing disorders. Acta Otolaryngol. 1996 Sep;116(5):666-71.

45. Gazquez I, Moreno A, Aran I, Soto-Varela A, Santos S, Perez-Garrigues $\mathrm{H}$, et al. MICA-STR A.4 is associated with slower hearing loss progression in patients with Meniere's disease. Otol Neurotol. 2012 Feb;33(2):223-9.

46. Requena T, Gazquez I, Moreno A, Batuecas A, Aran I, Soto-Varela A, et al. Allelic variants in TLR10 gene may influence bilateral affectation and clinical course of Meniere's disease. Immunogenetics. 2013 May;65(5):345-55.

47. Cabrera S, Sanchez E, Requena T, Martinez-Bueno M, Benitez J, Perez N, et al. Intronic variants in the NFKB1 gene may influence hearing forecast in patients with unilateral sensorineural hearing loss in Meniere's disease. PLoS One. 2014 Nov;9(11):e112171.

48. Frejo L, RequenaT, Okawa S, Gallego-Martinez A, Martinez-Bueno M, Aran I, et al. Regulation of Fn14 receptor and NF- $\mathrm{kB}$ underlies inflammation in Meniere's disease. Front Immunol. 2017 Dec;8: 1739.

49. XuWD, Zhao Y, Liu Y. Role of the TWEAK/Fn14 pathway in autoimmune diseases. Immunol Res. 2016 Feb;64(1):44-50.

50. Rask-Andersen H, Danckwardt-Lilliestrom N, Friberg U, House W. Lymphocyte-macrophage activity in the human endolymphatic sac. Acta Otolaryngol Suppl. 1991;485:15-7.

51. Okano T, Nakagawa T, Ito J. Distribution of bone marrow-derived cells in the vestibular end organs and the endolymphatic sac. Acta Otolaryngol Suppl. 2010 Nov;(563):88-94.

52. Moller MN, Kirkeby S, Vikesa J, Nielsen FC, Caye-Thomasen P. Gene expression in the human endolymphatic sac: the solute carrier molecules in endolymphatic fluid homeostasis. Otol Neurotol. 2015 Jun;36(5):915-22.

53. Moller MN, Kirkeby S, Vikesa J, Nielsen FC, Caye-Thomasen P. Expression of histamine receptors in the human endolymphatic sac: the molecular rationale for betahistine use in Menieres disease. Eur Arch Otorhinolaryngol. 2016 Jul;273(7):1705-10.

54. Hu BH, Zhang C, Frye MD. Immune cells and non-immune cells with immune function in mammalian cochleae. Hear Res. 2018 May;362:14-24.

55. O’Malley JT, Nadol JB Jr, McKenna MJ. Anti CD163+, Iba1+, and CD68+ cells in the adult human inner ear: normal distribution of an unappreciated class of macrophages/microglia and implications for inflammatory otopathology in humans. Otol Neurotol. 2016 
Jan;37(1):99-108

56. Liu W, Molnar M, Garnham C, Benav H, Rask-Andersen H. Macrophages in the human cochlea: saviors or predators: a study using super-resolution immunohistochemistry. Front Immunol. 2018 Feb; 9:223.

57. Kampfe Nordstrom C, Danckwardt-Lilliestrom N, Laurell G, LiuW, Rask-Andersen $\mathrm{H}$. The human endolymphatic sac and inner ear immunity: macrophage interaction and molecular expression. Front Immunol. 2019 Feb;9:3181.

58. Moller MN, Kirkeby S, Vikesa J, Nielsen FC, Caye-Thomasen P. Gene expression demonstrates an immunological capacity of the human endolymphatic sac. Laryngoscope. 2015 Aug;125(8):E269-75.

59. Broderick L. Hereditary autoinflammatory disorders: recognition and treatment. Immunol Allergy Clin North Am. 2019 Feb;39(1): 13-29.

60. Martinez-Quiles N, Goldbach-Mansky R. Updates on autoinflammatory diseases. Curr Opin Immunol. 2018 Dec;55:97-105.

61. Adams JC, Seed B, Lu N, Landry A, Xavier RJ. Selective activation of nuclear factor kappa B in the cochlea by sensory and inflammatory stress. Neuroscience. 2009 May;160(2):530-9.

62. Nie H, Zheng Y, Li R, Guo TB, He D, Fang L, et al. Phosphorylation of FOXP3 controls regulatory $\mathrm{T}$ cell function and is inhibited by TNF- $\alpha$ in rheumatoid arthritis. Nat Med. 2013 Mar;19(3):322-8.

63. Ren J, Li H, Lu Y.The determinations of tumor necrosis factor and interleukin 6 in serum of patients with sudden sensorineural hearing loss. Lin Chuang Er Bi Yan Hou Ke Za Zhi. 1998 Jul;12(7): 311-3.

64. Rahman MU, Poe DS, Choi HK. Etanercept therapy for immunemediated cochleovestibular disorders: preliminary results in a pilot study. Otol Neurotol. 2001 Sep;22(5):619-24.

65. Keithley EM, Wang X, Barkdull GC. Tumor necrosis factor alpha can induce recruitment of inflammatory cells to the cochlea. Otol Neurotol. 2008 Sep;29(6):854-9.

66. Wang X, Truong T, Billings PB, Harris JP, Keithley EM. Blockage of immune-mediated inner ear damage by etanercept. Otol Neurotol. 2003 Jan;24(1):52-7.

67. Lobo D, Trinidad A, Garcia-Berrocal JR, Verdaguer JM, RamirezCamacho R.TNFalpha blockers do not improve the hearing recovery obtained with glucocorticoid therapy in an autoimmune experimental labyrinthitis. Eur Arch Otorhinolaryngol. 2006 Jul;263(7): $622-6$

68. Kassner SS, Schottler S, Bonaterra GA, Stern-Strater J, Sommer U, Hormann K, et al. Proinflammatory and proadhesive activation of lymphocytes and macrophages in sudden sensorineural hearing loss. Audiol Neurootol. 2011;16(4):254-62.

69. Suslu N, Yilmaz T, Gursel B. Utility of immunologic parameters in the evaluation of Meniere's disease. Acta Otolaryngol. 2009 Nov; 129(11):1160-5.

70. Svrakic M, Pathak S, Goldofsky E, Hoffman R, Chandrasekhar SS, Sperling N, et al. Diagnostic and prognostic utility of measuring tumor necrosis factor in the peripheral circulation of patients with immune-mediated sensorineural hearing loss. Arch Otolaryngol Head Neck Surg. 2012 Nov;138(11):1052-8.

71. Pathak S, Stern C,Vambutas A. N-Acetylcysteine attenuates tumor necrosis factor alpha levels in autoimmune inner ear disease patients. Immunol Res. 2015 Dec;63(1-3):236-45.

72. Blesa S, Cortijo J, Mata M, Serrano A, Closa D, Santangelo F, et al. Oral $\mathrm{N}$-acetylcysteine attenuates the rat pulmonary inflammatory response to antigen. Eur Respir J. 2003 Mar;21(3):394-400.

73. Angeli SI, Abi-Hachem RN, Vivero RJ, Telischi FT, Machado JJ. L$\mathrm{N}$-Acetylcysteine treatment is associated with improved hearing outcome in sudden idiopathic sensorineural hearing loss. Acta Otolaryngol. 2012 Apr;132(4):369-76.

74. Thornberry NA, Bull HG, Calaycay JR, Chapman KT, Howard AD,
Kostura MJ, et al.A novel heterodimeric cysteine protease is required for interleukin-1 beta processing in monocytes. Nature. 1992 Apr; 356(6372):768-74.

75. Zheng D, LiwinskiT, Elinav E. Inflammasome activation and regulation: toward a better understanding of complex mechanisms. Cell Discov. 2020 Jun;6:36.

76. Andrei C, Margiocco P, Poggi A, Lotti LV,Torrisi MR, Rubartelli A. Phospholipases $\mathrm{C}$ and $\mathrm{A} 2$ control lysosome-mediated IL-1 beta secretion: implications for inflammatory processes. Proc Natl Acad Sci U S A. 2004;101(26):9745-50.

77. Sims JE, Smith DE.The IL-1 family: regulators of immunity. Nat Rev Immunol. 2010 Feb;10(2):89-102.

78. Pathak S, Vambutas A. Autoimmune inner ear disease patient-associated $28-\mathrm{kDa}$ proinflammatory $\mathrm{IL}-1 \beta$ fragment results from caspase7-mediated cleavage in vitro. JCI Insight. 2020 Feb;5(3):e130845.

79. Mandrup-Poulsen T, Pickersgill L, Donath MY. Blockade of interleukin 1 in type 1 diabetes mellitus. Nat Rev Endocrinol. 2010 Mar; 6(3):158-66.

80. Arend WP. Cytokine imbalance in the pathogenesis of rheumatoid arthritis: the role of interleukin-1 receptor antagonist. Semin Arthritis Rheum. 2001 Apr;30(5 Suppl 2):1-6.

81. Luheshi NM, Rothwell NJ, Brough D. Dual functionality of interleukin-1 family cytokines: implications for anti-interleukin-1 therapy. Br J Pharmacol. 2009 Aug;157(8):1318-29.

82. Ligumsky M, Simon PL, Karmeli F, Rachmilewitz D. Role of interleukin 1 in inflammatory bowel disease: enhanced production during active disease. Gut. 1990 Jun;31(6):686-9.

83. Gosselin D, Rivest S. Role of IL-1 andTNF in the brain: twenty years of progress on a Dr. Jekyll/Mr. Hyde duality of the innate immune system. Brain Behav Immun. 2007 Mar;21(3):281-9.

84. Hawkins PN, Lachmann HJ, McDermott MF. Interleukin-1-receptor antagonist in the Muckle-Wells syndrome. N Engl J Med. 2003 Jun;348(25):2583-4.

85. Hull KM, Shoham N, Chae JJ,Aksentijevich I, Kastner DL.The expanding spectrum of systemic autoinflammatory disorders and their rheumatic manifestations. Curr Opin Rheumatol. 2003 Jan;15(1): 61-9.

86. Pathak S, Goldofsky E, Vivas EX, Bonagura VR, Vambutas A. IL-1 $\beta$ is overexpressed and aberrantly regulated in corticosteroid nonresponders with autoimmune inner ear disease. J Immunol. 2011 Feb; 186(3):1870-9

87. Nakanishi H, Kawashima Y, Kurima K, Chae JJ, Ross AM, PintoPatarroyo G, et al. NLRP3 mutation and cochlear autoinflammation cause syndromic and nonsyndromic hearing loss DFNA34 responsive to anakinra therapy. Proc Natl Acad Sci U S A. 2017 Sep; 114(37):E7766-75.

88. Gattorno M, Martini A. Beyond the NLRP3 inflammasome: autoinflammatory diseases reach adolescence. Arthritis Rheum. 2013 May;65(5):1137-47.

89. Agostini L, Martinon F, Burns K, McDermott MF, Hawkins PN, Tschopp J. NALP3 forms an IL-1beta-processing inflammasome with increased activity in Muckle-Wells autoinflammatory disorder. Immunity. 2004 Mar;20(3):319-25.

90. Stojanov S, Kastner DL. Familial autoinflammatory diseases: genetics, pathogenesis and treatment. Curr Opin Rheumatol. 2005 Sep; 17(5):586-99.

91. Gattorno M, Tassi S, Carta S, Delfino L, Ferlito F, Pelagatti MA, et al. Pattern of interleukin-1beta secretion in response to lipopolysaccharide and ATP before and after interleukin-1 blockade in patients with CIAS1 mutations. Arthritis Rheum. 2007 Sep;56(9): 3138-48.

92. Church LD, Cook GP, McDermott MF. Primer: inflammasomes and interleukin 1beta in inflammatory disorders. Nat Clin Pract Rheumatol. 2008 Jan;4(1):34-42. 
93. Hashimoto S, Billings P, Harris JP, Firestein GS, Keithley EM. Innate immunity contributes to cochlear adaptive immune responses. Audiol Neurootol. 2005 Jan-Feb;10(1):35-43.

94. Henderson C, Goldbach-Mansky R. Monogenic IL-1 mediated autoinflammatory and immunodeficiency syndromes: finding the right balance in response to danger signals. Clin Immunol. 2010 May; 135(2):210-22.

95. MiraultT, Launay D, Cuisset L, Hachulla E, Lambert M, QueyrelV, et al. Recovery from deafness in a patient with Muckle-Wells syndrome treated with anakinra. Arthritis Rheum. 2006 May;54(5): 1697-700

96. Mantovani A, Bonecchi R, Martinez FO, Galliera E, Perrier P,Allavena $\mathrm{P}$, et al. Tuning of innate immunity and polarized responses by decoy receptors. Int Arch Allergy Immunol. 2003 Oct;132(2):109-15.

97. Vambutas A, Lesser M, Mullooly V, Pathak S, Zahtz G, Rosen L, et al. Early efficacy trial of anakinra in corticosteroid-resistant autoimmune inner ear disease. J Clin Invest. 2014 Sep;124(9):4115-22.

98. Frejo L, Gallego-Martinez A, Requena T, Martin-Sanz E, Amor-Dorado JC, Soto-Varela A, et al. Proinflammatory cytokines and response to molds in mononuclear cells of patients with Meniere disease. Sci Rep. 2018 Apr;8(1):5974.

99. Flook M, Frejo L, Gallego-Martinez A, Martin-Sanz E, Rossi-Izquierdo M, Amor-Dorado JC, et al. Differential proinflammatory signature in vestibular migraine and meniere disease. Front Immunol. 2019 Jun;10:1229.

100. Cai T, Jen HI, Kang H, Klisch TJ, Zoghbi HY, Groves AK. Characterization of the transcriptome of nascent hair cells and identification of direct targets of the Atoh1 transcription factor. J Neurosci. 2015 Apr;35(14):5870-83.

101. Honda K, Kim SH, Kelly MC, Burns JC, Constance L, Li X, et al. Molecular architecture underlying fluid absorption by the developing inner ear. Elife. 2017 Oct;6:e26851.

102. Elkon R, Milon B, Morrison L, Shah M,Vijayakumar S, Racherla M, et al. RFX transcription factors are essential for hearing in mice. Nat Commun. 2015 Oct;6:8549.

103. Hirano T. IL-6 in inflammation, autoimmunity and cancer. Int Immunol. 2021 Mar;33(3):127-48.

104. Winkles JA. The TWEAK-Fn14 cytokine-receptor axis: discovery, biology and therapeutic targeting. Nat Rev Drug Discov. 2008 May; 7(5):411-25.

105. Burkly LC. Regulation of tissue responses: theTWEAK/Fn14 pathway and otherTNF/TNFR superfamily members that activate noncanonical NFkB signaling. Front Immunol. 2015 Mar;6:92.

106. Baxter FO, Came PJ, Abell K, Kedjouar B, Huth M, Rajewsky K, et al. IKKbeta/2 induces TWEAK and apoptosis in mammary epithelial cells. Development. 2006 Sep;133(17):3485-94.

107. Park JS, Kwok SK, Lim MA, Oh HJ, Kim EK, Jhun JY, et al.TWEAK promotes osteoclastogenesis in rheumatoid arthritis. Am J Pathol. 2013 Sep;183(3):857-67.

108. Michaelson JS,Wisniacki N, Burkly LC, Putterman C. Role ofTWEAK in lupus nephritis: a bench-to-bedside review. J Autoimmun. 2012 Sep;39(3):130-42.

109. Serafini B, Magliozzi R, Rosicarelli B, Reynolds R, Zheng TS, Aloisi F. Expression of TWEAK and its receptor Fn14 in the multiple sclerosis brain: implications for inflammatory tissue injury. J Neuropathol Exp Neurol. 2008 Dec;67(12):1137-48.

110. Stephan D, Sbai O, Wen J, Couraud PO, Putterman C, Khrestchatisky M, et al. TWEAK/Fn14 pathway modulates properties of a human microvascular endothelial cell model of blood brain barrier. J Neuroinflammation. 2013 Jan;10:9.

111. Sun SC. Non-canonical NF-kB signaling pathway. Cell Res. 2011 Jan;21(1):71-85.

112. Ramadass V,VaiyapuriT,TergaonkarV. Small molecule NF-кB pathway inhibitors in clinic. Int J Mol Sci. 2020 Jul;21(14):5164.

113. Gilmore TD, Herscovitch M. Inhibitors of NF-kappaB signaling: 785 and counting. Oncogene. 2006 Oct;25(51):6887-99.

114. Ren Z, Wang L, Cui J, Huoc Z, Xue J, Cui H, et al. Resveratrol inhibits NF-kB signaling through suppression of p65 and IkappaB kinase activities. Pharmazie. 2013 Aug;68(8):689-94.

115. Crow MK, Ronnblom L. Type I interferons in host defence and inflammatory diseases. Lupus Sci Med. 2019 May;6(1):e000336.

116. Tanaka T, Narazaki M, Kishimoto T. IL-6 in inflammation, immunity, and disease. Cold Spring Harb Perspect Biol. 2014 Sep;6(10): a016295.

117. Tansey MG, Szymkowski DE. The TNF superfamily in 2009: new pathways, new indications, and new drugs. Drug DiscovToday. 2009 Dec;14(23-24):1082-8.

118. Lis K, Kuzawinska O, Balkowiec-Iskra E. Tumor necrosis factor inhibitors: state of knowledge. Arch Med Sci. 2014 Dec;10(6):117585.

119. Smolen JS, Landewe R, Breedveld FC, Buch M, Burmester G, Dougados $\mathrm{M}$, et al. EULAR recommendations for the management of rheumatoid arthritis with synthetic and biological disease-modifying antirheumatic drugs: 2013 update. Ann Rheum Dis. 2014 Mar; 73(3):492-509.

120. Dinarello CA. Overview of the IL-1 family in innate inflammation and acquired immunity. Immunol Rev. 2018 Jan;281(1):8-27.

121. Gabay C, Lamacchia C, Palmer G. IL-1 pathways in inflammation and human diseases. Nat Rev Rheumatol. 2010 Apr;6(4):232-41.

122. Ren K, Torres R. Role of interleukin-1beta during pain and inflammation. Brain Res Rev. 2009 Apr;60(1):57-64.

123. Dhimolea E. Canakinumab. MAbs. 2010 Jan-Feb;2(1):3-13.

124. Rincon M, Irvin CG. Role of IL-6 in asthma and other inflammatory pulmonary diseases. Int J Biol Sci. 2012;8(9):1281-90. 